\title{
Dirk Gevers
}

Over the past decade, researchers around the world have been getting serious about understanding how the microbes that live in and on us affect disease and health. Industry is now ramping up its investment into this space as well. Janssen, which has already partnered with the microbiome-focused biotechs Vedanta Biosciences and Second Genome, took its microbiome ambitions to the next level earlier this year by creating the Janssen Human Microbiome Institute. Heading the Institute is Dirk Gevers, a computational biologist who was formerly a group leader at the Broad Institute, in Cambridge, Massachusetts, USA, and a researcher on the Human Microbiome Project. The microbiome offers opportunity across therapeutic areas and drug modalities, he tells Asher Mullard.

Q. What's behind the growing interest of industry in microbiome-based drugs? I don't thinks it's due to a single breakthrough, but rather to a ripple effect of smaller breakthroughs that have really started to increase in amplitude.

In 2004-2005, sequencing technology was at the point where costs were dropping and we could start dabbling in new areas. The Human Genome Project was done, and people wanted to start on the next big thing. There was also this realization at that point about how many organisms live in and on us, and so researchers started turning to sequencing technology to explore these organisms.

From there, clinicians started to get interested. I spent a lot of time talking to clinicians and explaining what we were learning from the microbiome, and how we were applying new technologies to a very old field. I think what makes this field particularly exciting is that, very early on, people started thinking about how to translate findings into the clinic. I think that happened much faster with the microbiome than with the human genome, because whereas you are born with your genome, there was an early realization that you can change the microbiome.

The third big ripple has been pharma recognizing this and jumping in.

\section{Q. What's your aim with the Human Microbiome Institute?}

Janssen really recognized the potential of the microbiome many years ago. We just completed a review of activities across Janssen, and found that we already have two dozen different microbiome projects embedded across our different disease area research groups. All of this has really been happening through external collaborations. Our aim will now be to connect more external innovation with our internal expertise.

\section{Q. Which therapeutic areas offer quick} wins for microbiome-based medicines? A lot of research has happened in inflammatory bowel disease, and we have partnered with Vedanta to develop a cocktail of organisms that will be used to treat the disorder. Our goal is to get this product into clinical trials in more or less a year from now.

Type 1 diabetes is also emerging as a possible area, through some of the unbelievably large cohorts that have been built and studied over the past 10 years both in the United States and Europe. I feel that those data are going to be ripe for mining, and will indicate where the opportunities may lie.

There have also been studies on gut development in infants, and it looks like during the early years of development there really is an opportunity to shape the microbiome and modulate the likelihood of different diseases. Interesting studies have shown how breast-feeding affects the microbiome and impacts the risk of allergy, for example.

\section{Q. How do you think about the different} therapeutic modalities for microbiome-based drugs?

I basically see it in four bins.

Cocktails of organisms are bin number 1 , with Vedanta's product providing a good example. I see a lot of activity with far simpler cocktails as well, with researchers considering using just a few organisms or even just one organism as a potential product. This might be an early, more actionable area of research. You don't need to fully understand all the signals that the organisms are sending to the they are safe and effective.

The other bins really will come from deeper understanding of mode of action.

A second bin involves tapping into the signals - including metabolites - that are sent by the organisms in the microbiome. But these organisms are sending so many signals to the host that it is very early days to say "let's take this one signal and make it into a product". The way I've been thinking about this is that the host is basically a circuit board, and that multiple signals from the microbes help to set up the circuit board. If that circuit board is in the wrong setting, and you send or block one signal, you might not get any response. Or you might even get an incorrect response. We are still in the early days of understanding that, and host-microbe interaction is a very active research area.

A third bin involves understanding how signals are received by the host and which downstream pathways they trigger. You can then go back to more-traditional small molecules and biologics to make sure that the right pathways are switched on or off.

And then a fourth bin that needs to be explored is how you can train the immune system using vaccines to tolerate the right types of organisms or to push back against the wrong types of organisms. Some of the autoimmune diseases are basically an over-response of our immune system to organisms that other people can tolerate beautifully. So, how do you train the immune system better?

\section{Q And you'll work on diagnostics too?} We are still figuring out what makes a microbiome-based diagnostic reliable enough to be effective, but I strongly believe that the microbiome offers potential for diagnostics.

\section{Q What does the regulatory landscape look like for these therapies and diagnostics?} This is all new territory, not only for industry but also for regulators. At Janssen, we are looking to address and lead in this area, and our teams are really ramping up their expertise and interaction with the US Food and Drug Administration. 